\title{
Factors Affecting of Road Traffic Injuries in the Lower Southern Region of Thailand: A Content Analysis of Road Traffic Investigation Reports, 2006- 2019
}

Nuntaporn Klinjun ( $\nabla$ nuntaporn.kl@mail.wu.ac.th )

Walailak University

Matthew Kelly

Australian National University

Chanita Praditsathaporn

University of Phayao

Rewwadee Petsirasan

Walailak University

\section{Research Article}

Keywords: Road traffic injury, Risk factor, Road traffic investigation, Haddon matrix

Posted Date: December 28th, 2020

DOl: https://doi.org/10.21203/rs.3.rs-128534/v1

License: (c) (1) This work is licensed under a Creative Commons Attribution 4.0 International License.

Read Full License 
Factors affecting of road traffic injuries in the lower southern region of Thailand: a content analysis of road traffic investigation reports, 2006-2019

Nuntaporn Klinjun ${ }^{1, *}$, Matthew Kelly ${ }^{2}$, Chanita Praditsathaporn ${ }^{3}$, Rewwadee Petsirasan ${ }^{1}$

${ }^{1}$ School of Nursing, Walailak University, 222 Thai Buri, Tha Sala District, Nakhon Si

Thammarat, Thailand 80161

${ }^{2}$ Department of Global Health, Research School of Population Health, 62 Mills Road, Australian National University, Canberra ACT 0200

${ }^{3}$ School of Nursing, University of Phayao, 19 Moo2, Mae Ka, Mueang Phayao District, Phayao, Thailand 56000

* Correspondence: nuntaporn.k1@mail.wu.ac.th

\section{Highlights}

- The Haddon matrix can help identify factors (human, vehicle, physical and socio-economic) characteristics of road traffic injuries together with the temporal stages (pre-event, event, post-event).

- Human error-related factors injuries included driving with excessive speed, drowsiness and not having enough driving skill.

- Risks for passengers included not using seat belt, no self-defense, sitting in cargo area and the cab of the pickups, and being ejected from seats or vehicles. 


\begin{abstract}
Background - Thailand has the second highest rates of road traffic mortality globally. Detailed information on the combination of human, vehicle and environmental risks giving rise to each incident is important for addressing risk factors holistically. This paper presents the result of forensic road traffic investigation reports in Thailand and determines risk factor patterns for road traffic injuries.
\end{abstract}

Methods - Detailed forensic reports were extracted for 25 serious traffic accident events. For each report, accidents were characterized by the number of vehicles, number and type of injuries and deaths, road user types, involvement of roadside hazards, medical care provided, and human, vehicular and environmental influences on the incident. The Haddon matrix was used to analyze risk factors in three phases (pre-event, event, post-event) stratified by four agents (human, vehicle, physical and socio-economic environment).

Results - The 25 events analyzed involved 407 victims and 47 vehicles. $65.8 \%$ of victims were injured, including $22.0 \%$ who died. Median age of those injured was 27 years, and 28 years for fatalities. Vehicles crashing with fixed objects, and pickup-related crashes, accounted for the majority of accidents and deaths. Head or neck trauma was the main cause of death. The majority (61\%) of deaths occurred at the scene. Human error-related factors included speeding, and drowsiness from night and long-distance driving. Passenger risks included not using seat belt, sitting in cargo area and the cab of pickups. Not having anyone trained in first aid on the scene, first aid being provided by bystanders, and delayed calls to Emergency Medical Services increased injury risk. Overloaded vehicles, unsafe car modifications, no occupant safety equipment, and having unfixed seats were vehicular risks. Environmental risks included fixed objects on the roadside, no traffic lights, no guard rails, no traffic sign, road accident black spots, and hazardous objects roadside. 
Conclusions - Thailand must address all three temporal phases of the Haddon model and all three factors - human, vehicle and environment. At present traffic accidents cause much avoidable severe injury and death. The Haddon matrix is useful to structure road traffic investigations, revealing multi-level factors common on Thai roads.

Keywords: Road traffic injury, Risk factor, Road traffic investigation, Haddon matrix

\section{Introduction}

Road traffic injury is a significant cause of morbidity and mortality, and is in the top 10 causes of death globally, leading to approximately 1.4 million deaths each year. Among populations aged 15-29 years, road traffic injuries are the top cause of death [1]. Almost 75\% of road traffic accident deaths affect males aged under 25 years [2]. As well as mortality, road traffic injuries lead to significant morbidity, with global estimates of more than 20 million nonfatal road traffic injuries occurring each year. Disability and economic impacts of fatal and non-fatal injuries are substantial. As well as the age distribution of road traffic injury, socioeconomic status also plays a role in injury risk. Low- and middle-income countries experience $90 \%$ of road traffic accident mortality [2].

Southeast Asia is a particular hot spot for road traffic injury, accounting for $25 \%$ of global mortality, or more than 300,000 deaths annually [3] Within this region, Thailand has the highest rate of mortality from road traffic injury, and in 2015 had the second highest rate globally, following Libya, with an estimated fatality rate of around 36.2 per 100,000 population [4]. The incidence of traffic injuries rose from 449.0 per 100,000 in 2012 to 524.9 cases per 100,000 in 2016 [5].

As in many other countries Thai road traffic injury and road traffic mortality are connected to a number of identified and preventable risk factors. Firstly, motorcycles are associated with nearly $40 \%$ of all road traffic accidents. Male sex and being aged less than 40 years also increase risk of road traffic injury. Furthermore, recent surveys have shown that only 
around one-third of car users regularly wear safety belts [6], and regular helmet use on motorcycles was only just over 40\% [7].

National statistics such as those reported here however only give an overall view of the road traffic safety situation. Each road traffic incident has its own set of, generally preventable, drivers. Examining individual accident events in detail can help us understand where preventative measures could have been employed. The Haddon Matrix is a tool which can help to examine road traffic accidents in detail using a qualitative analysis method [8]. It allows the combined assessment of conditions leading to the accident, and the severity of injuries, in terms of human, vehicular, physical environment and socio-economic environment factors. This analytical method then adds a temporal dimension examining how these three factors operated pre-crash, during the crash, and post-crash.

The purpose of this paper is to use the Haddon Matrix approach to examine in detail a number of fatal and non-fatal injury incidents in Thailand and consider the appropriate injury prevention techniques. This information can be used to support the Thai government in implementing policies to reduce the number of road traffic injuries in Thailand. The objectives of this study were: 1 . To understand drivers of road traffic injuries in Thailand by using indepth road traffic investigations; and 2. To determine the factors influencing injury and injury severity using the Haddon's matrix model.

\section{Methods}

\subsection{Study design}

This study comprises a content analysis of road traffic investigation reports for traffic incidents occurring between November 2006 and April 2019 in the lower southern region of Thailand. The criteria for including incidents in the investigation are as follows: 1) Road traffic injuries resulting in five or more deaths, or 15 or more injuries; 2) Road traffic incidents at sites which have had injuries and deaths occur previously, more than 2-5 times in the same 
month; 3) Particularly noteworthy incidents from a community perspective, such as injuries to students, or accidents involving public transportation or ambulances [9] These reports were compiled by the reginal Office of Disease Prevention and Control 12, Songkhla Province (ODPC12), Department of Disease Control (DDC), Ministry of Public Health (MOPH), Thailand. This is the responsible government body for documenting road traffic injuries in this part of Southern Thailand. The content of these reports was analyzed using Haddon's matrix to understand the key characteristics of each incident.

\subsection{Data extraction and Filtering Criteria}

The first step of the study was to extract the full in-depth reports of selected road traffic injury incidents from the database of the reginal Office of Disease Prevention and Control 12, Songkhla Province, Thailand (ODPC12). The reports were prepared by multidisciplinary teams from the Ministry of Public Health including epidemiologists, nurses, public health officers, civil engineers, police and other related agencies. The reports included inspection outcomes from crash scenes, in-depth interviews with victims, eye-witnesses, and health staff, and information extracted from the medical records concerning ambulance and hospital treatment [10].

Recording forms were developed by the authors to extract the relevant variables from these reports for this study. Variables extracted comprised:

1. Event characteristics: vehicle-related crash and/or other parties involved in incident (such as pedestrians), involvement of roadside object hazards, collision types, and type of vehicle.

2. Road user information: sex, age-groups, road user type (driver, passenger, pedestrian), characteristics of those injured (driver, passenger, pedestrian), number of victims, number of injured, and number of deaths. 
3. Injury details: region of body injured consisted of head and neck, face, chest, abdomen and pelvis, extremities and pelvic girdle, and external and skin injuries. [11]

4. Prehospital trauma care: type of assistance provided, and mode of delivery to hospitals as reported by eyewitnesses, police officers, or rescue terms (First response unit: FR., Basic life support unit: BLS, Advanced life support unit: ALS)

5. Factors influencing the injury: this followed Haddon's matrix system addressing human, vehicle, physical environment, and socio-economic environment factors which influenced likelihood of injury within three phases of influence (pre-event, event, post-event) $[10,12,13]$

In total, 24 road traffic investigation reports were chosen with a total of 25 events because two events were included in the same report. These reports covered events in five provinces in the lower southern region of Thailand namely, Trang, Satun, Phatthalung, Songkhla, and Pattani.

\subsection{Data Analysis}

The data extraction was performed during April 2020. The first analysis step involved calculating descriptive statistics for the traffic incidents. Event characteristics, road user information, and injured body parts were described using frequencies and percentages.

Next the extracted content from the traffic accident reports was evaluated qualitatively using the Haddon's matrix model as an analytical framework. Factors influencing the likelihood and severity of injuries for each traffic accident were categorized in terms of the host, the vehicle, the physical environment and the socio-economic environment in three crucial temporal stages (the pre-event phase, the event phase and the post-event phase) [10, 14]. This analysis aims to identify determinants of road accidents themselves, and also the determinants of injury, injury severity and death, at multiple levels, and before, during and after 
the accident. The advantages of this approach are the ability to identify structural and behavioral risk factors.

\section{Results}

\subsection{Overview of event characteristics}

Table 1 shows the details of the 25 road traffic investigation reports analyzed, involving 407 victims, 47 vehicles, and 27 roadside object hazards. Of the victims, $268(65.8 \%)$ were injured, including 59 deaths (14.5\%). Roadside objects were a factor in 18 events. The incidents with the highest number of injuries were a multiple-vehicle crash in front of a school with 34 injured, followed by an overturned bus crashing into a tree with 24 injured. The incidents with the highest number of deaths were a school pickup crashing into a tree with 10 casualties, followed by a worker pickup running off the road and crashing into trees with 7 persons deceased. Events in which all victims died consisted of a car burning after hitting a tree, a prime mover truck crashing into a pedestrian bridge and a pickup crashing into a motorcycle.

Table 1 Traffic accident characteristics in terms of vehicle numbers, roadside object hazards, victims, injuries and deaths

\begin{tabular}{|c|c|c|c|c|c|}
\hline Events & Vehicles & $\begin{array}{c}\text { Roadside } \\
\text { objects }\end{array}$ & Victims & $\begin{array}{c}\text { Total Injured } \\
(\%)\end{array}$ & $\begin{array}{l}\text { Death } \\
(\%)^{*}\end{array}$ \\
\hline $\begin{array}{l}\text { 1. An overturned bus crashed into trees } \\
\text { after hitting a pedestrian }\end{array}$ & 1 & 2 trees & 26 & $17(64.5)$ & $2(7.7)$ \\
\hline $\begin{array}{l}\text { 2. A pickup crashed into other pickups } \\
\text { with the second pickup rolling and flipping } \\
\text { over a passenger }\end{array}$ & 3 & - & 18 & $18(100.0)$ & $2(11.1)$ \\
\hline $\begin{array}{l}\text { 3. A multiple-vehicle crash in front of a } \\
\text { school zone }\end{array}$ & 8 & 1 tree & 40 & $34(85.0)$ & $0(0.0)$ \\
\hline $\begin{array}{l}\text { 4. A school pickup crashing into a tree and } \\
\text { overturning after a car crashed into the } \\
\text { pickup }\end{array}$ & 2 & 1 tree & 20 & $18(90.0)$ & $1(5.0)$ \\
\hline $\begin{array}{l}\text { 5. A car crashing into a pickup and } \\
\text { running off the road, colliding with a } \\
\text { prime mover truck }\end{array}$ & 3 & 1 tree & 8 & $6(75.0)$ & $5(62.5)$ \\
\hline $\begin{array}{l}\text { 6. A chartered van crashing into trees and } \\
\text { overturning }\end{array}$ & 1 & 2 trees & 15 & $12(80.0)$ & $6(40.0)$ \\
\hline 7. An excursion bus overturned & 1 & - & 66 & $24(36.4)$ & $0(0.0)$ \\
\hline $\begin{array}{l}\text { 8. A car crashing into an ambulance, } \\
\text { which then crashed into a pickup }\end{array}$ & 3 & - & 7 & $2(28.6)$ & $0(0.0)$ \\
\hline 9. A car burning after hitting a tree & 1 & 1 tree & 4 & $4(100.0)$ & $\begin{array}{c}4 \\
(100.0)\end{array}$ \\
\hline
\end{tabular}




\begin{tabular}{|c|c|c|c|c|c|}
\hline Events & Vehicles & $\begin{array}{c}\text { Roadside } \\
\text { objects }\end{array}$ & Victims & $\begin{array}{c}\text { Total Injured } \\
\qquad \%)\end{array}$ & $\begin{array}{l}\text { Death } \\
(\%)^{*}\end{array}$ \\
\hline $\begin{array}{l}\text { 10. A saloon car crashing into an } \\
\text { electricity pole }\end{array}$ & 1 & $\begin{array}{l}1 \text { utility } \\
\text { pole }\end{array}$ & 9 & $9(100.0)$ & $6(66.7)$ \\
\hline $\begin{array}{l}11 \text { An armored pickup running off a road } \\
\text { crashing into trees and overturning }\end{array}$ & 1 & 3 trees & 2 & $2(100.0)$ & $\begin{array}{c}2 \\
(100.0)\end{array}$ \\
\hline $\begin{array}{l}\text { 12. An ambulance crashing into a car, } \\
\text { which then collided with pedestrians }\end{array}$ & 2 & - & 13 & $10(76.9)$ & $1(7.7)$ \\
\hline $\begin{array}{l}\text { 13. A school pickup running off a road and } \\
\text { crashing into a tree }\end{array}$ & 1 & 1 tree & 12 & $12(100.0)$ & $\begin{array}{c}10 \\
(83.3)\end{array}$ \\
\hline 14. A car burning after hitting a tree & 1 & 1 tree & 3 & $3(100.0)$ & $2(66.7)$ \\
\hline $\begin{array}{l}\text { 15. A prime mover truck crashing into a } \\
\text { pedestrian bridge }\end{array}$ & 1 & $\begin{array}{l}1 \text { concrete } \\
\text { pillar }\end{array}$ & 1 & $1(100.0)$ & $\begin{array}{c}1 \\
(100.0)\end{array}$ \\
\hline 16. A car hitting a tree & 1 & 1 tree & 2 & $2(100.0)$ & $1(50.0)$ \\
\hline $\begin{array}{l}\text { 17. A pickup truck running off a road and } \\
\text { crashing into a tree and a utility pole }\end{array}$ & 1 & $\begin{array}{l}1 \text { tree } \\
1 \text { utility } \\
\text { pole }\end{array}$ & 4 & $4(100.0)$ & $3(75.0)$ \\
\hline $\begin{array}{l}\text { 18. An excursion pickup public transport } \\
\text { running off a road and crashing into trees }\end{array}$ & 1 & 2 trees & 21 & $21(100.0)$ & $2(9.5)$ \\
\hline $\begin{array}{l}19 \text { A pickup public transport pickup } \\
\text { running off a road and crashing into a } \\
\text { utility pole }\end{array}$ & 1 & $\begin{array}{l}1 \text { utility } \\
\text { pole }\end{array}$ & 5 & $5(100.0)$ & $1(20.0)$ \\
\hline 20. A pickup crashing into a motorcycle & 2 & 1 tree & 2 & $1(50.0)$ & $1(50.0)$ \\
\hline $\begin{array}{l}\text { 21. An excursion bus crashing into the } \\
\text { back of another excursion bus, crashing } \\
\text { into the back of a pickup }\end{array}$ & 3 & Non & 81 & $18(22.2)$ & $0(0.0)$ \\
\hline $\begin{array}{l}\text { 22. A pickup truck crashing a public van, } \\
\text { running off a road and hitting trees }\end{array}$ & 2 & 2 trees & 15 & $14(93.3)$ & $1(6.7)$ \\
\hline $\begin{array}{l}23 \text { A car u-turned crossed into the opposite } \\
\text { lane and crashing a worker pickup truck, } \\
\text { overturning and ran off the road }\end{array}$ & 2 & non & 18 & $18(100.0)$ & $0(0.0)$ \\
\hline $\begin{array}{l}\text { 24. An ambulance crashing into the back } \\
\text { of a prime mover truck }\end{array}$ & 2 & non & 4 & $3(75.0)$ & $1(25.0)$ \\
\hline $\begin{array}{l}\text { 25. A truck crashing a worker pickup } \\
\text { truck, running off a road and hitting two } \\
\text { trees and a utility pole }\end{array}$ & 2 & $\begin{array}{l}2 \text { trees } \\
1 \text { utility } \\
\text { pole }\end{array}$ & 11 & $10(90.9)$ & $7(63.6)$ \\
\hline Total & 47 & 27 & 407 & $\begin{array}{c}268 \\
(65.8)\end{array}$ & $\begin{array}{c}59 \\
(14.5)\end{array}$ \\
\hline
\end{tabular}

*Percentage of traffic incident victims who died

The time of occurrence of road traffic injures in the 25 events are shown in table 2 . The most accidents occurred between midday and 3 p.m. The most injuries occurred in accidents between $3 \mathrm{pm}$ and $6 \mathrm{pm}, 36.9 \%$ of total injured and $27.1 \%$ of road traffic fatalities.

Table 2 Number of events, total injured and death in road traffic injuries by time of day

\begin{tabular}{lcccccc}
\hline \multicolumn{1}{c}{ Time of day } & $\begin{array}{c}\text { Number } \\
\text { (25 events) }\end{array}$ & $\mathbf{\%}$ & $\begin{array}{c}\text { Total injured } \\
\text { (No=268) }\end{array}$ & \% & $\begin{array}{c}\text { Death } \\
\text { (No=59) }\end{array}$ & \% \\
\hline Midnight to 3.00 a.m. & 0 & 0.0 & 0 & 0.0 & 0 & 0.0 \\
3.01 a.m. to 6.00 a.m. & 4 & 16.0 & 34 & 12.7 & 13 & 22.0 \\
6.01 a.m. to 9.00 a.m. & 3 & 12.0 & 31 & 11.6 & 10 & 16.9 \\
9.01 a.m. to Midday & 3 & 12.0 & 31 & 11.6 & 3 & 5.1
\end{tabular}


Midday to 3.00 p.m. 3.01 p.m. to 6.00 p.m. 28.0

2

Table 3 shows approximately $52.0 \%(n=13)$ of events were single-vehicle accidents.

The most common types of single-vehicle accidents were crashes with fixed objects such as a tree, or electric pole, 7 events $(53.8 \%) .72 \%(n=18)$ of all events involved fixed object crashes.

Table 3 Number and percent of collision types

\begin{tabular}{lcc}
\hline \multicolumn{1}{c}{ Collision types } & $\begin{array}{c}\text { Number } \\
\text { (25 events) }\end{array}$ & $\boldsymbol{\%}$ \\
\hline single-vehicle crashes & $\mathbf{1 3}$ & $\mathbf{5 2 . 0}$ \\
Overturned car, fire & 0 & 0.0 \\
Involving pedestrians & 0 & 0.0 \\
Fixed object crashes such as a tree, or a utility pole & 7 & 53.8 \\
Fixed object crashes and crashes involving pedestrians or overturned, fire & 6 & 46.2 \\
\hline Two-vehicle crashes & $\mathbf{8}$ & $\mathbf{3 2 . 0}$ \\
Overturned car, fire & 3 & 37.5 \\
involving pedestrians & 1 & 12.5 \\
Fixed object crashes such as a tree, or a utility pole & 3 & 37.5 \\
Fixed object crashes and crashes involving pedestrians or non-fixed objects & 1 & 12.5 \\
\hline Multi-vehicle crashes & $\mathbf{4}$ & $\mathbf{1 6 . 0}$ \\
Overturned car, fire & 0 & 0.0 \\
Crashes involving pedestrians & 0 & 0.0 \\
Fixed object crashes such as a tree, or a utility pole & 0 & 0.0 \\
Fixed object crashes and crashes involving pedestrians or non-fixed objects & 2 & 50.0 \\
\hline
\end{tabular}

\subsubsection{Type of vehicles}

Of the 47 vehicles in total, there was $17(36.2 \%)$ vehicles whose occupants were injured. The maximum vehicle number involved in a single event was eight vehicles. Figure 1a shows approximately $42.6 \%(n=20)$ of vehicles involved in accidents were pick-ups, and $17.0 \%(n=8)$ were cars. Figure $1 \mathrm{~b}$ shows the distribution of vehicle types was similar when we looked only at vehicles whose occupants were injured, approximately $42.4 \%(n=14)$ of vehicle type involving injury were pick-ups, and $21.2 \%(\mathrm{n}=7)$ cars. 


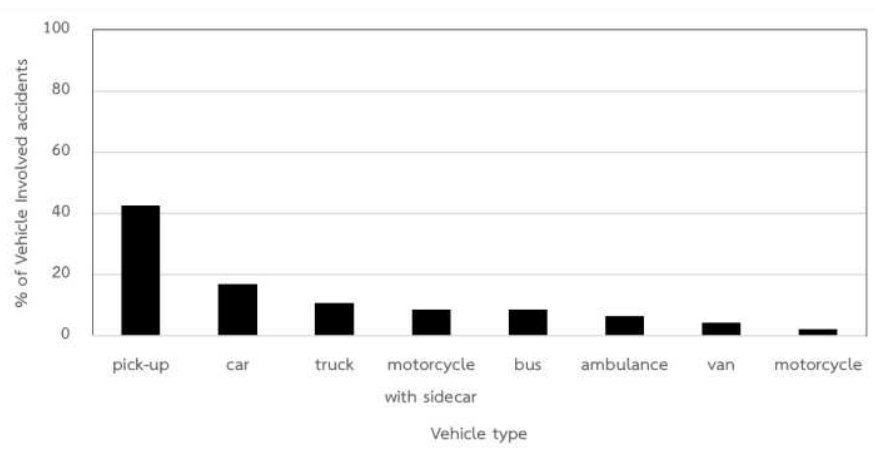

a

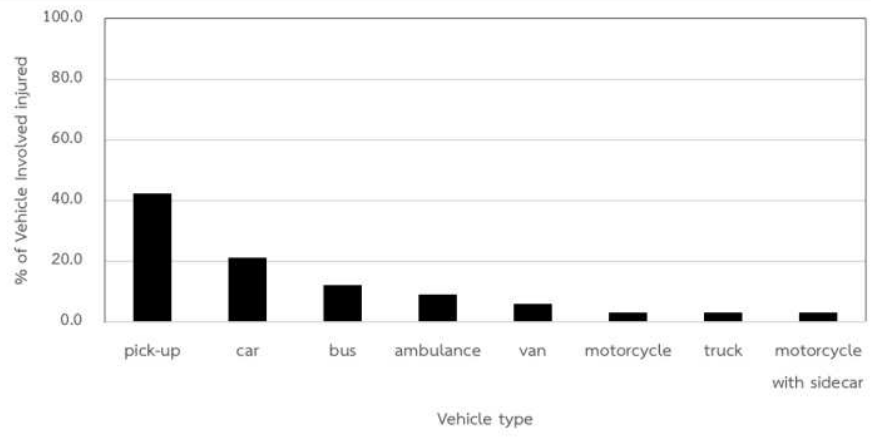

b

Figure 1: Frequency of vehicle type involved in accidents among: (a) the total 47 vehicles and (b) 33 vehicles where injury occurred

\subsection{Demographic data of road users}

Table 4 presents females as 1.6 times more likely to be injured than men. The median age of those injured was 27 years 5 months (range: 3 months- -88 years). Case fatality rate was $22.0 \%(n=59), 1.2$ times more women than men died. The median age of the deceased was 28 years (range: 3 months- 82 years). The age distribution of injuries and deaths showed the highest number occurred among those aged less than 15 years, accounting for 63 (11.9\%) injuries and accounting for $10(16.9 \%)$ deaths. The highest case fatality rate (CFR) was in the age-group 40-44 years (38.1\%). The vehicles associated with the most casualties were pickups accounting for $133(51.6 \%)$ injuries and 28 (49.1\%) deaths.

Table 4 Demographic details of road traffic injury victims by number and percentage

\begin{tabular}{|c|c|c|c|c|c|}
\hline \multirow[b]{2}{*}{ Demographic data of road users } & \multicolumn{2}{|c|}{ Total injured } & \multicolumn{2}{|c|}{ Death } & \multirow{2}{*}{$\begin{array}{l}\text { CFR } \\
(\%)\end{array}$} \\
\hline & $\begin{array}{c}\text { Number } \\
(n=268)\end{array}$ & $\%$ & $\begin{array}{c}\text { Number } \\
(n=59)\end{array}$ & $\%$ & \\
\hline \multicolumn{6}{|l|}{ Sex } \\
\hline Female & 165 & 61.6 & 32 & 54.2 & 19.4 \\
\hline Male & 103 & 38.4 & 27 & 45.8 & 26.2 \\
\hline Female: Male & \multicolumn{2}{|c|}{ 1:1.6 } & \multicolumn{2}{|c|}{$1: 1.2$} & \\
\hline \multicolumn{6}{|l|}{ Age-groups (year) } \\
\hline$<15$ & 63 & 24.9 & 10 & 16.9 & 15.9 \\
\hline $15-19$ & 27 & 10.7 & 8 & 13.6 & 29.6 \\
\hline $20-24$ & 22 & 8.7 & 3 & 5.1 & 13.6 \\
\hline
\end{tabular}




\begin{tabular}{|c|c|c|c|c|c|}
\hline \multirow[b]{2}{*}{ Demographic data of road users } & \multicolumn{2}{|c|}{ Total injured } & \multicolumn{2}{|c|}{ Death } & \multirow[b]{2}{*}{$\begin{array}{l}\text { CFR } \\
(\%)\end{array}$} \\
\hline & $\begin{array}{c}\text { Number } \\
(n=268)\end{array}$ & $\%$ & $\begin{array}{c}\text { Number } \\
(\mathrm{n}=59)\end{array}$ & $\%$ & \\
\hline $25-29$ & 25 & 9.9 & 9 & 15.3 & 36.0 \\
\hline $30-34$ & 16 & 6.3 & 3 & 5.1 & 18.8 \\
\hline $35-39$ & 9 & 3.6 & 3 & 5.1 & 33.3 \\
\hline $40-44$ & 21 & 8.3 & 8 & 13.6 & 38.1 \\
\hline $45-49$ & 18 & 7.1 & 5 & 8.5 & 27.8 \\
\hline $50-54$ & 18 & 7.1 & 4 & 6.8 & 22.2 \\
\hline $55-59$ & 11 & 4.3 & 3 & 5.1 & 27.3 \\
\hline$\geq 60$ & 23 & 9.1 & 3 & 5.1 & 13.0 \\
\hline Total & 253 & 100.0 & 59 & 100.0 & 23.3 \\
\hline Mean age (year) & \multicolumn{2}{|c|}{$\begin{array}{c}31.1 \\
3 \text { months }\end{array}$} & \multicolumn{2}{|c|}{ 32.0 } & \\
\hline Minimum age (year) & & \multicolumn{2}{|c|}{3 months } & \\
\hline Maximum age (year) & & & & & \\
\hline \multicolumn{6}{|l|}{ Vehicle used by casualties } \\
\hline Motorcycle & 1 & 0.4 & 1 & 1.7 & 100.0 \\
\hline Motorcycle with sidecar & 2 & 0.8 & 0 & 0.0 & 0.0 \\
\hline Car & 28 & 10.8 & 18 & 31.6 & 64.3 \\
\hline Pick-up & 133 & 51.6 & 28 & 49.1 & 21.1 \\
\hline Van & 26 & 10.1 & 7 & 17.5 & 26.9 \\
\hline Ambulance & 8 & 3.1 & 1 & 12.3 & 12.5 \\
\hline Bus & 58 & 22.5 & 1 & 1.7 & 1.7 \\
\hline Truck & 2 & 0.4 & 1 & 1.7 & 50.0 \\
\hline Total & 258 & 100.0 & 57 & 100.0 & 22.1 \\
\hline
\end{tabular}

\subsubsection{Injury by role in accident}

Table 5 shows that the most of the casualties were passengers, comprising $229(85.5 \%)$ injuries and $46(78.0 \%)$ deaths. This group was followed by drivers, comprising $29(10.8 \%)$ injuries and $11(18.6 \%)$ deaths. The highest case fatality rate (CFR) was found among drivers $37.9 \%$

Table 5 Number and percent of total injured, death and CFR by type of road user

\begin{tabular}{lccccc}
\hline \multirow{2}{*}{ Types of injured } & \multicolumn{2}{c}{ Total injured $(\mathbf{n = 2 6 8})$} & \multicolumn{2}{c}{ Death $(\mathbf{n = 5 9 )}$} & \multirow{2}{*}{ CFR (\%) } \\
\cline { 2 - 5 } & Number & $\mathbf{\%}$ & Number & $\mathbf{\%}$ & \\
\hline Driver & 29 & 10.8 & 11 & 18.6 & 37.9 \\
Passenger & 229 & 85.5 & 46 & 78.0 & 20.1 \\
Pedestrian & 10 & 3.7 & 2 & 3.4 & 20.0 \\
\hline
\end{tabular}

\subsubsection{Place of deaths}

Figure 2 presents places of deaths. The majority of deaths were death at scene 39 persons $(66.1 \%)$, follow by death in a hospital 16 persons $(27.1 \%)$ 


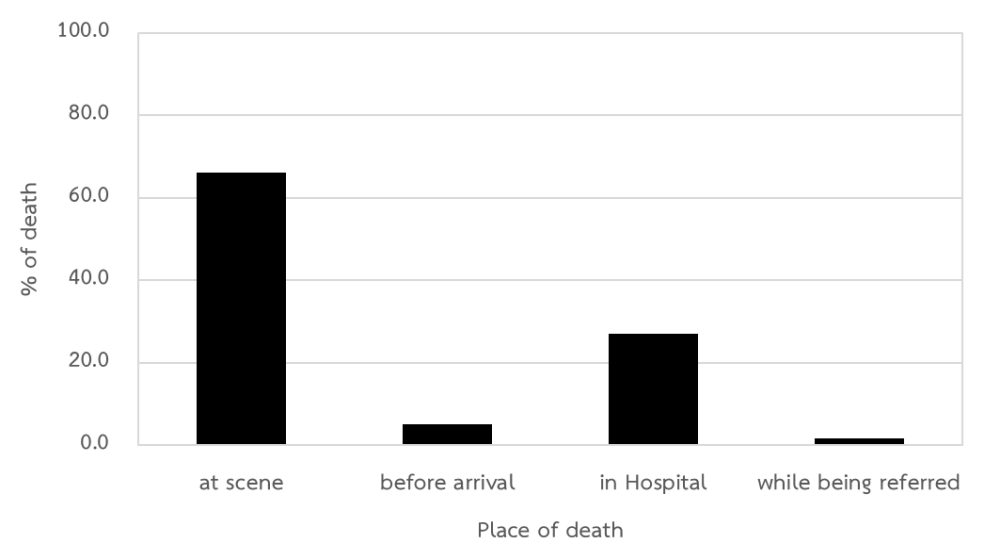

Figure 2: Bar chart percent of deaths by places of deaths

\subsection{Body region of injuries}

One person could be injured in several body parts. Table 6 shows that the body parts affected amongst those injured and body parts involved in fatal injuries. The most frequently injured body regions were extremities and pelvic girdle, 118 (44.0\%) injuries, followed by head/neck 111 (41.4\%) injuries. The most frequently injured body regions in fatal accidents were head/neck, $45(76.3 \%)$ accidents, followed by extremities and pelvic girdle $29(49.1 \%)$.

Table 6 Distribution of body regions injured in the 25 events (multiple response)

\begin{tabular}{lcccc}
\hline \multicolumn{1}{c}{$\begin{array}{c}\text { Body region of injuries } \\
\text { (multiple response) }\end{array}$} & \multicolumn{2}{c}{ Total injuries } & \multicolumn{2}{c}{ Death } \\
\cline { 2 - 5 } & No & \% & No & \% \\
\hline Head/neck (include middle inner ear) & 111 & 41.4 & 45 & 76.3 \\
Face (include eye balls) & 51 & 19.0 & 10 & 16.9 \\
Thorax (Chest) & 58 & 21.6 & 21 & 35.6 \\
Abdominal or pelvic contents & 46 & 17.1 & 11 & 18.6 \\
Extremities or pelvic girdle & 118 & 44.0 & 29 & 49.1 \\
External and body surface & 95 & 35.4 & 19 & 32.2 \\
\hline
\end{tabular}

\subsection{Prehospital care system}

Table 7 shows that the first call in the scene in the case of a serious injury accident was call to Medical Emergency Call from 1669 (44.0\%). 
Table 7 Number and percent of 25 events by source of call after crashing

\begin{tabular}{lrr}
\hline \multicolumn{1}{c}{ Source of call } & Number & \% \\
\hline Police (191) & 9 & 36.0 \\
Medical Emergency Call (1669) & 11 & 44.0 \\
District Hospital/FR in the area & 5 & 20.0 \\
Total & 25 & 100.0 \\
\hline
\end{tabular}

Table 8 shows the transportation from scene had many teams or many bystanders in each event. 84 percent of the injured persons had been transferred to the hospital by first response units: FR and 76 percent of these persons were transferred by hospital EMS.

Table 8 Number and percent of the mode of transportation of the injured from scene (multiple response)

\section{The transportation from scene (multiple transportations)}

\section{Bystander}

Hospit

al

EMS unit (FR) (BLS/

1. An overturned bus crashed into trees after hitting a ALS

Total pedestrian

2. A pickup crashed into other pickups before the second pickup rolling and flipping over a passenger

3. A multiple-vehicle crash in front of a school zone

4. A school pickup crashing into a tree and overturning after a car crashed the pickup

5. A car crashing a pickup and running off the road, colliding with a prime mover truck

6. A chartered van crashing trees and overturning

7. An excursion bus overturned

8. A car crashing an ambulance, crashing a pickup

9. A car burning after hitting a tree

10. A saloon car crashing a utility pole

11 An armored pickup running off a road crashing trees and overturning

12. An ambulance crashing a car, crashing sex pedestrians

13. A school pickup running off a road and crashing into a tree

14. A car burning after hitting a tree

15. A prime mover truck crashing a pedestrian bridge

16. A car hitting a tree

17. A pickup truck running off a road and crashing into a tree and a utility pole

18. An excursion pickup public transport running off a road and crashing into trees

19 A pickup public transport pickup running off a road and crashing into a utility pole

20. A pickup crashing into a motorcycle

21. An excursion bus crashing into the back of another excursion bus, crashing into the back of a pickup

\begin{tabular}{|c|c|c|}
\hline$\checkmark$ & $\checkmark$ & 2 \\
\hline$\checkmark$ & & 3 \\
\hline$\checkmark$ & $\checkmark$ & 3 \\
\hline$\checkmark$ & $\checkmark$ & 3 \\
\hline$\checkmark$ & $\checkmark$ & 2 \\
\hline$\checkmark$ & $\checkmark$ & 3 \\
\hline$\checkmark$ & $\checkmark$ & 3 \\
\hline & $\checkmark$ & 1 \\
\hline$\checkmark$ & & 1 \\
\hline$\checkmark$ & $\checkmark$ & 2 \\
\hline & & 2 \\
\hline$\checkmark$ & $\checkmark$ & 2 \\
\hline$\checkmark$ & $\checkmark$ & 3 \\
\hline$\checkmark$ & $\checkmark$ & 2 \\
\hline & $\checkmark$ & 11 \\
\hline$\checkmark$ & & 1 \\
\hline$\checkmark$ & $\checkmark$ & 4 \\
\hline$\checkmark$ & $\checkmark$ & 2 \\
\hline$\checkmark$ & & 1 \\
\hline$\checkmark$ & $\checkmark$ & 2 \\
\hline
\end{tabular}




\begin{tabular}{|c|c|c|c|c|c|}
\hline $\begin{array}{l}\text { The transportation from scene } \\
\text { (multiple transportations) }\end{array}$ & Bystander & $\begin{array}{l}\text { Police } \\
\text { officer }\end{array}$ & $\begin{array}{l}\text { First } \\
\text { response } \\
\text { unit (FR) }\end{array}$ & $\begin{array}{c}\text { Hospit } \\
\text { al } \\
\text { EMS } \\
\text { (BLS/ } \\
\text { ALS) }\end{array}$ & Total \\
\hline $\begin{array}{l}\text { 22. A pickup truck crashing a public van, running off a } \\
\text { road and hitting trees }\end{array}$ & & & $\checkmark$ & $\checkmark$ & 2 \\
\hline $\begin{array}{l}23 \text { A car u-turned crossed into the opposite lane and } \\
\text { crashing a worker pickup truck, overturning and ran off } \\
\text { the road }\end{array}$ & & & $\checkmark$ & $\checkmark$ & 2 \\
\hline $\begin{array}{l}\text { 24. An ambulance crashing into the back of a prime } \\
\text { mover truck }\end{array}$ & & & $\checkmark$ & $\checkmark$ & 2 \\
\hline $\begin{array}{l}25 \text {. A truck crashing a worker pickup truck, running off } \\
\text { a road and hitting two trees and a utility pole }\end{array}$ & & & $\checkmark$ & $\checkmark$ & 2 \\
\hline Total & $\begin{array}{c}4 \\
(16.0 \%)\end{array}$ & $\begin{array}{c}8 \\
(32.0 \%)\end{array}$ & $\begin{array}{c}21 \\
(84.0 \%)\end{array}$ & $\begin{array}{c}19 \\
(76.0 \%)\end{array}$ & $\begin{array}{c}52 \\
(100.0 \%)\end{array}$ \\
\hline
\end{tabular}

\subsection{Risk factors}

More than half of the accidents overall involved four risk factors, 14 events (56.0\%), followed by three risk factors, 10 events $(40.0 \%)$. All accidents involved a combination of human error in conjunction with physical environment risk factors. Vehicle defects were a risk factor in $84.0 \%$ of accidents. All road traffic accidents which caused injury involved a combination of human errors in conjunction with vehicle defects. Physical environment defects were a factor in $96.0 \%$ of injury causing accidents.

Table 9 shows a Haddon's Matrix analysis of risk factors in three crucial stages of the road traffic injuries. In the pre-event phase, speeding was the most common human error, accounting for 17 events (68.0\%). Fixed objects in safety zone was a physical environment defect for 15 events $(60.0 \%)$, and accident black spots were a social-economic environment defect involved in 12 events (48.0\%). In the event phase, the human error of not using the safety belt and being ejected from the seats or the vehicles, was most common in causing road traffic injuries. Vehicle hitting fixed objects in safety zones was found among most road traffic injuries. In the post-event phase, delayed call to EMS and no persons trained in first aid on scene were found to be the largest contributor to injury risk. 'An additional table 9 file shows this in more detail [see Supplementary table 1]'. 


\section{Discussion}

The reviews of 25 in-depth road injury investigations presented here reveal some of the common patterns and risk factors connected to the major problem of road injuries in Thailand. The Haddon Matrix approach provided a useful tool to examine the multiple factors connected to injury and severity of injury in physical and temporal dimensions. This adds to much previous literature focusing on mainly driver behavior or other singular risks. This study showed that multiple injuries and more serious injuries most often involved vehicles leaving the road and collisions with roadside hazards. This pattern has also been found in other indepth road traffic analyses $[15,16,17]$. Moreover, the pickup was the most common vehicle type in road traffic accidents and was associated with the most injuries and deaths. Severity of injuries and fatalities were also linked to the number of occupants in the vehicles, as found elsewhere [18], and carrying passengers in the cab and cargo area $[19,20]$. In fatal accidents, most victims died at the scene. Head or neck trauma was the main cause of deaths [21, 22].

The results of the study confirmed other research showing that road traffic injuries are caused by the main risk factors comprising human errors, vehicle defects and environment defects $[13,23,24,25]$. The work of multidisciplinary road traffic investigation teams were important keys to recognizing human errors increasing risk pre-crash including behaviors of driver (speeding, drowsiness from night or long-distance driving, insufficient driving skills), and risk behaviors of others (insufficient understanding of traffic rules, risk behaviors of pedestrians or passengers). This study confirmed that exceeding the speed limit was the most common violation involved in road accidents [24] and lead to more severe injuries [26, 27]. Moreover, the drowsy driver appears widespread and has been found in several countries [28, 29].

The vehicle defects causing the pre-crash consisted mainly of overloading and modification. The environment defects pre-event were documented as many fixed objects in 
safety zone, no traffic lights, no guard rails, no traffic signs. It was also worth noting that there have been frequent crashes at these accident blackspot areas. This study is similar to observations in other studies $[10,16,30]$. However, this study showed that multiple risk factors at the pre-crash phase lead to the severity of traffic accidents at that time more than any single risk factor such as "high speed in the presence of overloading and fixed objects in safety zone", "high speed while distracted in spite of overloading and fixed objects in safety zone", "high speed in spite of drowsy from night driving and long-distance driving together with overloading and fixed objects in safety zone", " high speed while bent down to pick up things on floor", "Distracted driving from impaired shoes which go against the accelerator while U-turn"

Based on the in-depth road traffic injury investigation in crash risk factors, the human errors involved non use of seat belt, sitting in a place without safety device such as the cargo area and the cab of the pickups, and being ejected from seats or vehicles. Some other event factors were documented such as no occupant's safety belt in vehicle, vehicle structural damage, collision with object on and off road, the body impacted ground or fixed objects on side road. This study is similar to publications, and observations in other studies $[10,16,17$, $31,32,33]$. Then, multiple inter-related risk factors at the crash phase lead to the severity of traffic injuries such as combinations of: "non use of seat belt, the body collision with object on vehicle or ejected from vehicle and hit into the tree", or "the pickups rolled over and hit the tree, passenger were sitting in the cargo area of the pickups, no self-defense, ejected from the cargo", "the pickups ran off the road hitting the tree making the passenger were sitting in the cab of the pickups that no seat belt clashing with object on vehicle"

Post-crash a further set of risks affected severity of injury. The important post-crash risk factors were reported as not having any persons trained in first aid at the scene, delayed call to EMS, or non-availability of EMS. Fatality and disability resulting from accidents was also associated with other post-event factors including being stuck in vehicle, flames in the car, vehicle over 
the body, difficult to access and evacuate, and lack of slitting equipment. This phase is important to reduce fatalities and disabilities [10, 25].

Searching for in-depth information on all elements of the road injury is important for finding the root cause of the problem, where the immediate injury may be just the tip of the iceberg. $[34,35,36]$. The analyses presented in this paper provide multiple avenues to improve road safety in Thailand. These include improvements in human risk behaviors, the improvement of physical road infrastructure, safety standards for vehicles, higher rates of first aid training among the general population, and better coordination of EMS services. A holistic approach to road traffic accident prevention in Thailand can both reduce the injury rate and the severity of injuries incurred.

\section{Availability of data and materials}

The datasets used and analyzed during this study are available from the corresponding author follow by reasonable request.

\section{Abbreviations}

ODPC12: The reginal Office of Disease Prevention and Control 12, Songkhla Province, Thailand

DDC: Department of Disease Control

MOPH: Ministry of Public Health

FR: First response unit

BLS: Basic life support unit

ALS: Advanced life support unit

EMS: Emergency medical services

\section{References}

1. Chen S, Kuhn M, Prettner K, Bloom DE. The global macroeconomic burden of road injuries: estimates and projections for 166 countries. The Lancet Planetary Health. 2019 
Sep 1;3(9): e390-8.

2. World Health Organization. Road traffic injuries: key facts. Geneva: WHO; 2020. Available from https://www.who.int/health-topics/road-safety\#tab=tab 1. Cited 26 Oct 2020

3. Kraonual S, Lim A, Ueranantasun A, Kakchapati S. Patient and injury characteristics associated with road traffic mortality in general hospitals in southern Thailand. Asian Biomedicine. 2019 Dec 19;13(2):71-7.

4. Jomnonkwao S, Uttra S, Ratanavaraha V. Forecasting road traffic deaths in Thailand: Applications of time-series, curve estimation, multiple linear regression, and path analysis models. Sustainability. 2020 Jan;12(1):395.

5. Suphanchaimat R, Sornsrivichai V, Limwattananon S, Thammawijaya P. Economic development and road traffic injuries and fatalities in Thailand: an application of spatial panel data analysis, 2012-2016. BMC public health. 2019 Dec 1;19(1):1449. https://doi.org/10.1186/s12889-019-7809-7

6. Vallibhakara SA, Plitponkarnpim A, Suriyawongpaisal P, Thakkinstian A. The Nationwide Surveillance of Seat Belt Usage and Encouraging Factors of Increasing the Seat Belt Rate in Thailand: A Road Safety Survey. JOURNAL OF THE MEDICAL ASSOCIATION OF THAILAND. 2018 Jun 1;101(6):809-19.

7. Suriyawongpaisa P, Thakkinstian A, Rangpueng A, Jiwattanakulpaisarn P, Techakamolsuk P. Disparity in motorcycle helmet use in Thailand. International journal for equity in health. 2013 Dec 1;12(1):74.

8. Haddon W. The changing approach to the epidemiology, prevention, and amelioration of trauma: the transition to approaches etiologically rather than descriptively based. Injury Prevention. 1999 Sep 1;5(3):231-5.

9. Waiyanate, N. Road traffic injury investigation. Nonthaburi: Bureau of Epidemiology, 
Department of Disease Control, Ministry of Public Health; 2006 (In Thai)

10. Klinjun N, Chinwong D, Sleigh A. Epidemiology of Multiple Casualty Incidents from Road Accidents in Thailand, 2006-2011. OSIR Journal. 2017 Dec 27;10(4):1-8.

11. Ministry of Public Health. Epidemiology division. Condense Chart (AIS 85). Bangkok: MOPH; 1995 (In Thai)

12. Barnett DJ, Balicer RD, Blodgett D, Fews AL, Parker CL, Links JM. The application of the Haddon matrix to public health readiness and response planning. Environmental health perspectives. 2005 May;113(5):561-6.

13. Mohan D, Khayesi M, Tiwari G, Nafukho FM. Road traffic injury prevention training manual. World Health Organization; 2006.

14. Williams, AF. The Haddon matrix: its contribution to injury prevention and control. In: NATIONAL CONFERENCE ON INJURY PREVENTION AND CONTROL, 3RD, 1999, BRISBANE, QUEENSLAND, AUSTRALIA. 2000.

15. Koushki P, Al-Kandari F. Road safety: Prioritization of roadside hazard improvement. KUWAIT JOURNAL OF SCIENCE AND ENGINEERING. 2006 Dec 1;33(2):147.

16. Boontob N, Ponboon S, Aniwattakulchai P, Anurakamolkul C, Kanitpong K. In-Depth Accident Aanalysis due to Roadside Hazard: Thailand Case Study. In Proceedings of the Eastern Asia Society for Transportation Studies Vol. 7 (The 8th International Conference of Eastern Asia Society for Transportation Studies, 2009) 2009 (pp. 414-414). Eastern Asia Society for Transportation Studies.

17. Solah MS, Ariffin AH, Isa MH, Wong SV. In-depth crash investigation on bus accidents in Malaysia. Journal of Society for Transportation and Traffic Studies. 2013;3(1):22-31.

18. Chang LY, Mannering F. Analysis of injury severity and vehicle occupancy in truck-and non-truck-involved accidents. Accident Analysis \& Prevention. 1999 Sep 1;31(5):579-92. 19. Agran P, Winn D, Anderson C. Injuries to occupants in cargo areas of pickup trucks. 
Western journal of medicine. 1994 Nov;161(5):479.

20. Anderson CL, Agran PF, Winn DG, Greenland S. Fatalities to occupants of cargo areas of pickup trucks. Accident Analysis \& Prevention. 2000 Jul 1;32(4):533-40.

21. Birgani AG, Hakim A, Zare K. Epidemiologic study of fatal traffic accidents in Khuzestan province in in 2010. Scientific Journal of Rescue \& Relief. 2012;4(2):28-35.

22. Sadeghi-Bazargani H, Samadirad B, Shahedifar N, Golestani M. Epidemiology of road traffic injury fatalities among car users; a study based on forensic medicine data in East Azerbaijan of Iran. Bulletin of Emergency \& Trauma. 2018 Apr;6(2):146.

23. Runyan CW. Introduction: back to the future-revisiting Haddon's conceptualization of injury epidemiology and prevention. Epidemiologic reviews. 2003 Aug 1;25(1):60-4.

24. Masoumi K, Forouzan A, Barzegari H, Darian AA, Rahim F, Zohrevandi B, Nabi S. Effective factors in severity of traffic accident-related traumas; an epidemiologic study based on the Haddon matrix. Emergency. 2016;4(2):78-82.

25. Bachani AM, Peden M, Gururaj G, Norton R, Hyder AA. Road traffic injuries. In: Charles NM, Rachel N, Olive K, Kirk RS, editors. Injury Prevention and Environmental Health. $3^{\text {rd }}$ ed. The World Bank; 2017. p. 35-54.

26. Copsey N, Drupsteen L, Kampen JV, Kuijt-Evers L, Schmitz-Felten E, Verjans M. A review of accidents and injuries to road transport drivers. Luxembourg: Publications Office of the European Union, 2010. Available from https://osha.europa.eu/en/publications/review-accidents-and-injuries-road-transport-drivers. Cited 26 Oct 2020

27. Zhang G, Yau KK, Chen G. Risk factors associated with traffic violations and accident severity in China. Accident Analysis \& Prevention. 2013 Oct 1;59: 18-25.

28. Connor J, Norton R, Ameratunga S, Robinson E, Civil I, Dunn R, Bailey J, Jackson R. Driver sleepiness and risk of serious injury to car occupants: population based case 
control study. Bmj. 2002 May 11;324(7346):1125.

29. Nabi H, Guéguen A, Chiron M, Lafont S, Zins M, Lagarde E. Awareness of driving while sleepy and road traffic accidents: prospective study in GAZEL cohort. Bmj. 2006 Jul $6 ; 333(7558): 75$.

30. Somchainuck O, Taneerananon P, Jaritngam S. An in-depth investigation of roadside crashes on Thai National Highways. Engineering Journal. 2013;17(2):63-74.

31. Kloeden CN, McLean AJ, Baldock MR, Cockington AJ. Severe and fatal car crashes due to roadside hazards. NHMRC Road Accident Research Unit The University of Adelaide. 1999 May.

32. Luathep P, Taneerananon S, Thongchim P, Mama S, Somchainuek O. Roadside Crash: A Tragic Lesson from Thailand. In Proceedings of the Eastern Asia Society for Transportation Studies Vol. 7 (The 8th International Conference of Eastern Asia Society for Transportation Studies, 2009) 2009 (pp. 413-413). Eastern Asia Society for Transportation Studies.

33. Hoque MS, Hasan MR. Involvement of vehicle factors in road accidents. Journal of Civil Engineering (IEB). 2007 Jun;35(1):17-27.

34. Wilks J, Watson B. Road safety and international visitors in Australia: looking beyond the tip of the iceberg. Travel Medicine International. 1998;16(5):194-8.

35. Jacobs G, Aeron-Thomas A, Astrop A. Estimating global road fatalities. 2000. Available from http://trl.demo.varistha.co.uk/uploads/trl/documents/TRL445.pdf. Cited 26 Oct 2020

36. Castro C, García-Fernández P, Ventsislavova P, Esiman E, Crundall D. 887 Analysing the offender driver behaviour: recidivism, just the tip of the Iceberg?. 2016. Available from https://injuryprevention.bmj.com/content/injuryprev/22/Suppl 2/A316.2.full.pdf.

Cited 26 Oct 2020

\section{Acknowledgements}


The authors would like to thank the staff of the reginal Office of Disease Prevention and Control 12, Songkhla Province, Thailand. We also thank all of agencies and the people who provided information on the accident investigations.

\section{Funding}

This work was supported by Walailak University, Thailand, an individual scholarship [grant number WU-IRG-63-043]. The funder has no role all of the study design, data collection and data analysis or a policy maker.

\section{Author information}

\section{Affiliations}

School of Nursing, Walailak University, 222 Thai Buri, Tha Sala District, Nakhon Si

Thammarat, Thailand

Nuntaporn Klinjun

Department of Global Health, Research School of Population Health, 62 Mills Road, Australian National University, Canberra, Australia

Matthew Kelly

School of Nursing, University of Phayao, 19 Moo2, Mae Ka, Mueang Phayao District, Phayao, Thailand

Chanita Praditsathaporn

School of Nursing, Walailak University, 222 Thai Buri, Tha Sala District, Nakhon Si

Thammarat, Thailand

Rewwadee Petsirasan

\section{Contributions}

Conceptual framework, Methodology, Data management, Content analysis, NK; Content analysis, Validation, MK, CP and RP; Writing-Original draft, NK; Validation, Writingreview and editing MK; All authors have read and approved the final manuscript. 
Corresponding author

Correspondence to Nuntaporn Klinjun

\section{Ethics declarations}

\section{Ethics approval and consent to participate}

As all the data were secondary data of the full in-depth investigation reports which were no individual information, no names, or identifying information and did not track back to each person in each events of accident. Permission to use the information and submit for publication was granted by the reginal Office of Disease Prevention and Control 12, Songkhla Province, Thailand. We had strictly followed the confidential requirement. The data is not linked to any individual subjects and does not identify any subjects. However, all protocols were approved by Human Research Ethics Committee of Walailak university with approval number: WUEC19-234-01.

\section{Consent for publication}

Not applicable.

\section{Availability of data and materials}

The authors confirm that the secondary data supporting of this study are available within the manuscript. The road traffic investigation reports were by the reginal Office of Disease Prevention and Control 12, Songkhla Province, Thailand. The reports that support this study are available on request from the corresponding author.

\section{Competing interests}

The authors declare that they have no competing interests. 
Figures

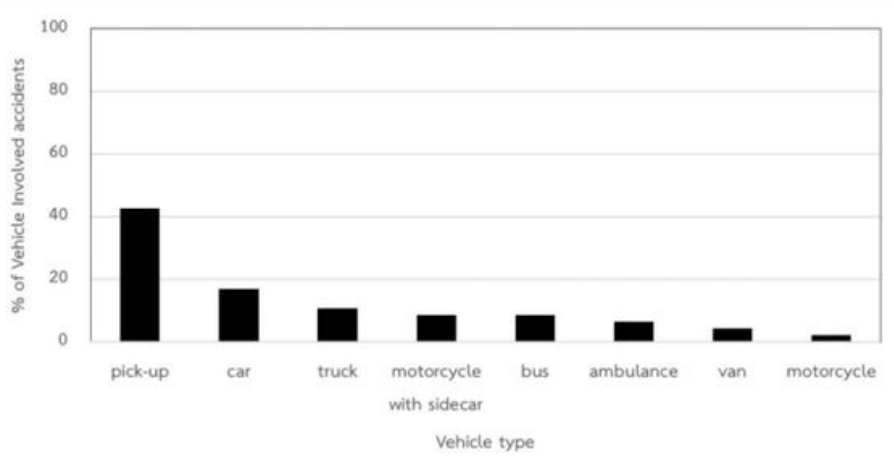

a

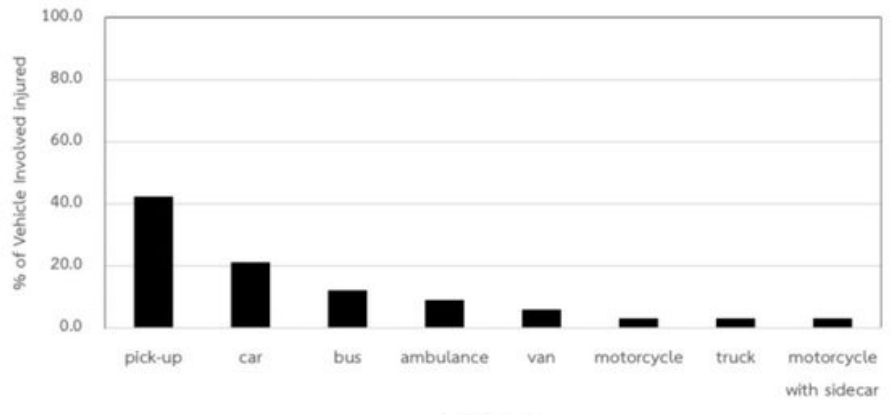

Vehicle type

b

\section{Figure 1}

Frequency of vehicle type involved in accidents among: (a) the total 47 vehicles and (b) 33 vehicles where injury occurred

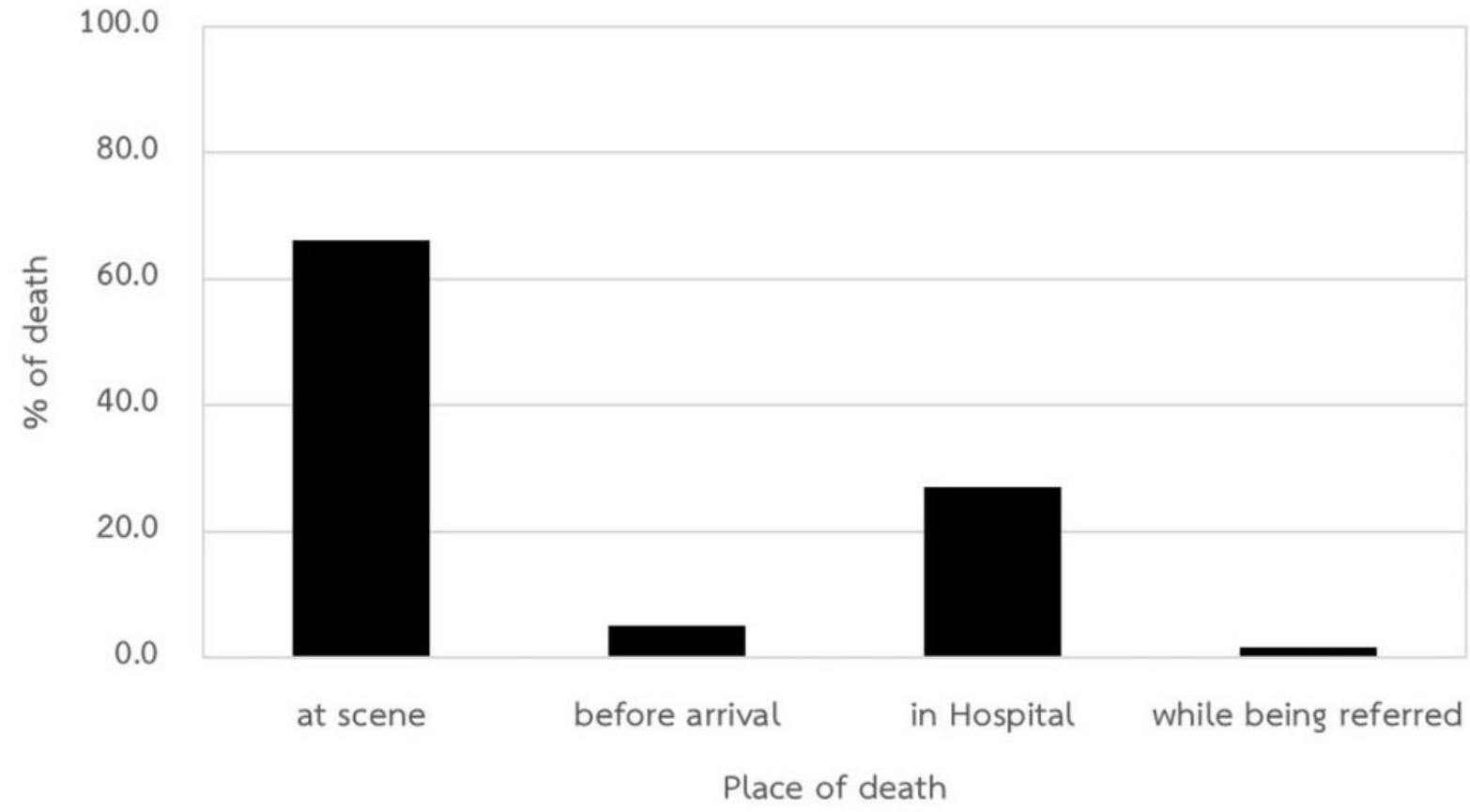

Figure 2

Bar chart percent of deaths by places of deaths

\section{Supplementary Files}


This is a list of supplementary files associated with this preprint. Click to download.

- Supplementarytable1.docx 